\title{
RELAXED GRAIN CLUSTER (RGC) HOMOGENIZATION SCHEME
}

\author{
D.D. Tjahjanto* P. Eisenlohr, F. Roters
}

\author{
Max-Planck-Insitut für Eisenforschung, Düsseldorf, Germany
}

\begin{abstract}
An efficient homogenization scheme for polycrystals is presented. The scheme is based on a generalization of the grain interaction (GIA) model. A volume element consisting of eight $(=2 \times 2 \times 2)$ hexahedral grains is considered. The kinematics of the relaxed grain cluster (RGC) scheme is formulated within a finite deformation framework, where the relaxation of the local deformation gradient of each individual grain is described by interface relaxation vectors. In the present model, the relaxation vectors are determined such that the total energy (or work) density of the cluster is minimum. A penalty term is added into the energy minimization landscape, which accounts for an energy density associated to the mismatch at the grain boundaries due to relaxations. Effectively, this penalty term mimics the kinematical condition of deformation compatibility at the grain boundaries. Furthermore, simulations have been performed for a polycrystalline sample. The overall behavior of the sample undergoing uniaxial tension and simple shear, is studied for various microstructural configurations. The prediction of the RGC scheme is compared with predictions using other averaging schemes, as well as the result of finite element (FE) simulation.
\end{abstract}

KEYWORDS: Grain interaction, Polycrystalline plasticity, Homogenization method

\section{INTRODUCTION}

A homogenization or coarse-graining scheme plays a vital role in multiscale modeling and simulations, particularly, in translating the detailed response of the material constitutive models at lower scales into the effective response required at higher scales. There are two opposing aspects that need to be addressed in homogenization issues, namely, (i) to maximize the quality of the overall prediction by preserving all relevant information from lower scale mechanisms, yet (ii) to minimize the amount of computational effort. In the context of polycrystalline metals, various homogenization schemes have been proposed in the last few decades, ranging from the most simple assumptions (i.e., Taylor and Sachs models), semianalytical grain interaction-based models (see e.g., [1-3]), up to complex numerical techniques (see e.g., $[4,5]$ ).

The present work is aimed at developing an efficient homogenization scheme, called the relaxed grain cluster (RGC) model. This model is based on the generalization of the grain cluster concept (see e.g., [1, 2]), which focussed on close interactions (in terms of deformations and stresses) between neighboring grains.

\section{RELAXED GRAIN CLUSTER MODEL}

\subsection{DEFORMATION AND RELAXATIONS}

Let $\overline{\boldsymbol{x}}$ be a macro-scale material point in the reference configuration and $\overline{\boldsymbol{F}}$ the macro-scale deformation gradient that maps the point $\overline{\boldsymbol{x}}$ into a point $\overline{\boldsymbol{y}}$ in the deformed configuration. In the present model, the material point $\overline{\boldsymbol{x}}$

\footnotetext{
*Corresponding author: Max-Planck-Str. 1, 40237 Düsseldorf, Ger-
} many. Phone: +49(211)6792959. Email: d.tjahjanto@mpie.de

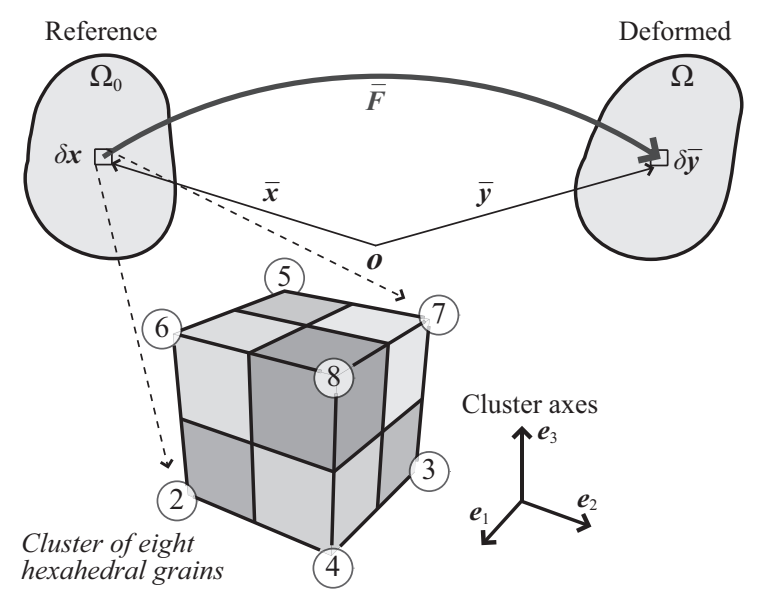

Figure 1: Macro-scale body in the reference and deformed configurations, where a material point is represented by a cluster of eight hexahedral grains.

is represented by a cluster of eight $(=2 \times 2 \times 2)$ hexahedral grains, as shown in Fig. 1. Furthermore, the (local) deformation gradient $\boldsymbol{F}^{g}$ of grain $g$ can be expressed by

$$
\boldsymbol{F}^{g}=\overline{\boldsymbol{F}}+\frac{1}{v_{0}^{g}} \sum_{\alpha=1}^{3}\left(\boldsymbol{a}_{\alpha}^{g} \otimes \boldsymbol{n}_{\alpha}^{g}+\boldsymbol{a}_{-\alpha}^{g} \otimes \boldsymbol{n}_{-\alpha}^{g}\right),
$$

where $\boldsymbol{a}_{\alpha}^{g}$ is a relaxation vector describing the relative displacement of face $\alpha$ of grain $g$ with respect to the overall deformation, with $\boldsymbol{n}_{\alpha}^{g}$ a unit vector normal to the face $\alpha$ (see Fig, 2 top), and $v_{0}^{g}$ measures the volume fraction of grain $g$ in the reference configuration. The relaxation vectors $\boldsymbol{a}_{\alpha}^{g}$ provide additional degrees of freedom to the full constraints (FC) Taylor assumption, where $\boldsymbol{F}^{g}=\overline{\boldsymbol{F}}$. 


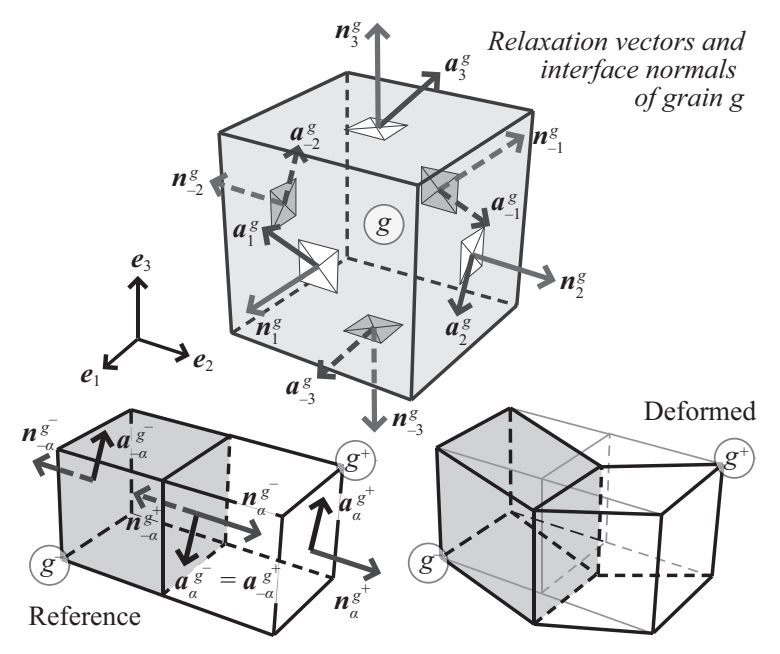

Figure 2: Relaxation vectors applied at six faces of grain $g$ (top). The effect of a relaxation vector at the shared interface between grains $g^{-}$and $g^{+}$, respectively (bottom).

Consider two neighboring grains $g^{-}$and $g^{+}$as shown in Fig. 2 bottom, where face $\alpha$ of grain $g^{-}$is identical to face $-\alpha$ of grain $g^{+}$. Consequently, the relaxation vector at this interface must be the same, i.e.,

$$
\boldsymbol{a}_{-\alpha}^{g^{+}}=\boldsymbol{a}_{\alpha}^{g^{-}} \quad \text { and } \quad \boldsymbol{a}_{\alpha}^{g^{+}}=\boldsymbol{a}_{-\alpha}^{g^{-}},
$$

where the second relation in (2) is derived from the assumption of a periodic grain arrangement. In order to eliminate rigid-body translations from the effective deformation of the aggregate, the sum of the relaxation vectors must vanish. In the case of the two grain aggregate, this condition leads to

$$
\boldsymbol{a}_{-\alpha}^{g}=-\boldsymbol{a}_{\alpha}^{g}
$$

The overall deformation gradient $\overline{\boldsymbol{F}}$ is computed by the volumetric average of the local deformation gradients as

$$
\overline{\boldsymbol{F}}=\sum_{g=1}^{8} v_{0}^{g} \boldsymbol{F}^{g} .
$$

\subsection{MISMATCH AND PENALTY ENERGY}

In the $2 \times 2 \times 2$-grain cluster, the relaxation of an interface may cause incompatibility at other interfaces (i.e., cross-effect), as illustrated in Fig. 3. Introduce a tensor $\boldsymbol{N}_{\alpha}^{g}$ that measures the mismatch at interface $\alpha$ of grain $g$. Assuming a planar interface, where the deformation gradient varies only in the direction normal to the interface, the mismatch tensor $\boldsymbol{N}_{\alpha}^{g^{-}}$is computed as

$$
\boldsymbol{N}_{\alpha}^{g^{-}}=-\left(\boldsymbol{n}_{\alpha}^{g^{-}} \times\left(\boldsymbol{G}_{\alpha}^{g^{-}}\right)^{T}\right)^{T}
$$

where $\boldsymbol{G}_{\alpha}^{g^{-}}=\frac{1}{2}\left(\boldsymbol{F}^{g^{+}}-\boldsymbol{F}^{g^{-}}\right)$, which measures the jump of deformation gradient across the interface zone $\left(g^{-}, \alpha\right)$. Furthermore, define $\mathcal{R}_{\alpha}^{g}$ as an energy density associated with the mismatch, defined as

$$
\mathcal{R}_{\alpha}^{g}=\frac{1}{2} \mu^{g} \sinh \left(c_{0}\left\|\boldsymbol{N}_{\alpha}^{g}\right\|\right)
$$

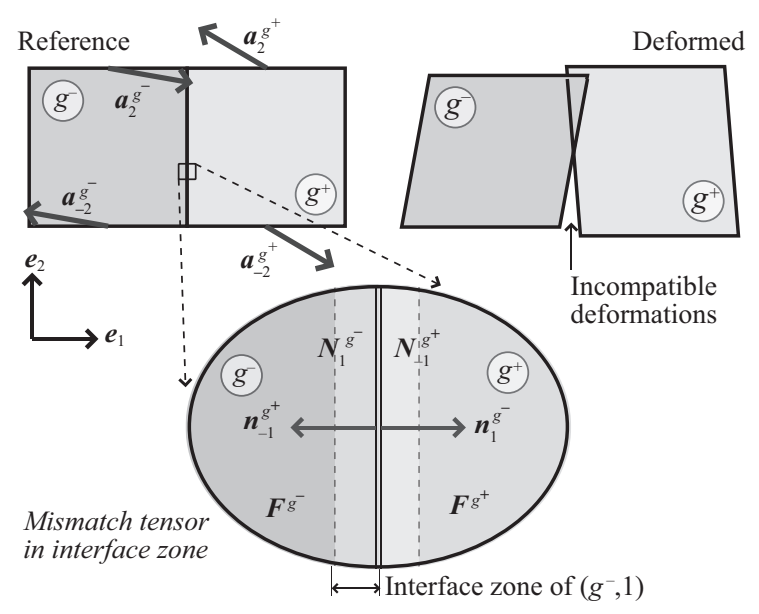

Figure 3: Mismatch at the interface due to side-effect of relaxation of other interfaces. The amount of mismatch is equally shared by the neighboring grains.

with $\mu^{g}$ the (equivalent) shear modulus of grain $g, c_{0}$ a scalar that determines the over-proportionality of the energy density function (6), and $\left\|\boldsymbol{N}_{\alpha}^{g}\right\|=\left(\boldsymbol{N}_{\alpha}^{g} \cdot \boldsymbol{N}_{\alpha}^{g}\right)^{1 / 2}$ the magnitude of the mismatch. The overall mismatch energy density can be computed as

$$
\overline{\mathcal{R}}=\sum_{g=1}^{8} v_{0}^{g} \mathcal{R}^{g}=\sum_{g=1}^{8} v_{0}^{g} \sum_{\alpha=1}^{3}\left(\mathcal{R}_{\alpha}^{g}+\mathcal{R}_{-\alpha}^{g}\right) .
$$

\subsection{STRESS AND ENERGY MINIMIZATION}

Let $\boldsymbol{P}^{g}$ be the first Piola-Kirchhoff stress of grain $g$, which is energetically conjugated to the deformation gradient $\boldsymbol{F}^{g}$. The stress $\boldsymbol{P}^{g}$ is related to $\boldsymbol{F}^{g}$ via the grain constitutive model. The effective stress and work (or constitutive deformation energy) density of the cluster are computed as the volumetric average of the corresponding local quantities as

$$
\overline{\boldsymbol{P}}=\sum_{g=1}^{8} v_{0}^{g} \boldsymbol{P}^{g} \text { and } \overline{\mathcal{W}}=\sum_{g=1}^{8} v_{0}^{g} \mathcal{W}^{g},
$$

with $\boldsymbol{P}^{g}$ and $\mathcal{W}^{g}$, respectively, the stress and work density of grain $g$. The evolution of the local work density follows $\dot{\mathcal{W}}^{g}=\boldsymbol{P}^{g} \cdot \dot{\boldsymbol{F}}^{g}$. The relaxation vectors $\boldsymbol{a}_{\alpha}^{g}$ are computed such that the total energy density, given by,

$$
\overline{\mathcal{E}}=\overline{\mathcal{W}}+\xi_{0} \overline{\mathcal{R}}
$$

is minimum, where $\xi_{0}$ determines the net-contribution or weight of the mismatch energy density, which is accounted for as a penalty. The solution of the above minimization problem is obtained by the stationary points, where $\partial \overline{\mathcal{E}} / \partial \boldsymbol{a}_{\alpha}^{g}=\mathbf{0}$, for all interfaces $\alpha= \pm 1,2,3$ of all grains $g=1, \ldots, 8$. Using the chain-rule to evaluate the partial derivatives $\partial \overline{\mathcal{E}} / \partial \boldsymbol{a}_{\alpha}^{g}$, the criteria for energy minimum is equivalent to the condition of stress equilibrium at (interior) interfaces between two neighboring grains $g^{-}$ and $g^{+}$, as

$$
\left(\boldsymbol{P}^{g^{+}}+\boldsymbol{R}^{g^{+}}\right) \boldsymbol{n}_{-\alpha}^{g^{+}}+\left(\boldsymbol{P}^{g^{-}}+\boldsymbol{R}^{g^{-}}\right) \boldsymbol{n}_{\alpha}^{g^{-}}=\mathbf{0},
$$


with $\boldsymbol{n}_{-\alpha}^{g^{+}}=-\boldsymbol{n}_{\alpha}^{g^{-}}$. In expression (10), $\boldsymbol{R}_{\alpha}^{g}$ stands for a stress-like penalty term, which is obtained as

$\boldsymbol{R}_{\alpha}^{g}=\xi_{0} \sum_{\beta=1}^{3} \frac{\lambda_{\beta}^{g} c_{0}}{\left\|\boldsymbol{N}_{\beta}^{g}\right\|}\left(\left(\boldsymbol{N}_{\beta}^{g}\right)^{T} \times \boldsymbol{n}_{\beta}^{g}\right)^{T} \cosh \left(c_{0}\left\|\boldsymbol{N}_{\beta}^{g}\right\|\right)$

where $\lambda_{\beta}^{g}=\frac{1}{2}\left(\mu^{g}+\mu^{g_{\beta}^{\star}}\right)$, with $g_{\beta}^{\star}$ the grain sharing the interface $\beta$ with grain $g$.

As a summary, for any given $\overline{\boldsymbol{F}}$, the local deformation gradients $\boldsymbol{F}^{g}$ can be constructed from the relaxation vectors $\boldsymbol{a}_{\alpha}^{g}$ using (1). In the case of the $2 \times 2 \times 2$-grain cluster and observing the kinematical restrictions (2) and (3), there are 12 independent relaxation vectors to be determined. In order to determine these vectors, a Newton-Raphson iteration procedure is employed for solving the set of nonlinear equations (10), i.e., the stress equilibrium condition for all 12 interior interfaces. A more detailed description of the RGC model can be found elsewhere [6].

\section{ELEMENTARY SIMULATIONS}

\subsection{SINGLE CRYSTAL MODEL PARAMETERS}

A classical single crystal elasto-plasticity model for facecentered cubic metals is used as the local constitutive behavior of the individual grains, in accordance with the model proposed by Kalidindi, et al. [7]. Table 1 lists the material parameters used in this model and the assigned values.

Table 1: Values of material parameters in single crystal elasto-plasticity model.

\begin{tabular}{lll}
\hline Model parameter(s) & Value(s) & Unit \\
\hline \hline Orthotropic elastic moduli & $\mathbb{C}_{11}=149.5$ & $\mathrm{GPa}$ \\
(Voigt's notation) & $\mathbb{C}_{12}=72.5$ & $\mathrm{GPa}$ \\
& $\mathbb{C}_{44}=45.3$ & $\mathrm{GPa}$ \\
Equivalent shear modulus & $\mu=42.6$ & $\mathrm{GPa}$ \\
Reference slip rate & $\dot{\gamma}_{0}=0.001$ & $\mathrm{~s}^{-1}$ \\
Rate-dependency exponent & $m=0.02$ & \\
Initial slip resistance & $s_{0}=56.0$ & $\mathrm{MPa}$ \\
Slip resistance saturation & $s_{\infty}=224.0$ & $\mathrm{MPa}$ \\
Initial hardening modulus & $h_{0}=600$ & $\mathrm{MPa}$ \\
Hardening exponent & $a_{s}=4.0$ & \\
Latent hardening ratio & $q=1.4$ & \\
\hline
\end{tabular}

In terms of the " 313 "-Euler rotation angles (Bunge convention), the orientations of the constituent grains $g=1, \ldots, 8$ with respect to the cluster coordinate system are given by, respectively, $\left(\varphi_{1} ; \phi ; \varphi_{2}\right)=(0.0 ; 18.4 ; 9.0)$, $(38.6 ; 0.0 ; 0.0), \quad(12.7 ; 45.0 ; 0.0), \quad(25.7 ; 45.0 ; 35.3)$, $(19.3 ; 26.6 ; 0.0),(32.1 ; 26.6 ; 24.1),(6.4 ; 45.0 ; 19.5)$, and $(45.0 ; 33.7 ; 15.5)$, all angles are in degree.

\subsection{GRAIN CLUSTER RESPONSE}

In the present paper, the RGC scheme is used to simulate the response of a polycrystalline sample subjected to the two loading conditions of (i) uniaxial tension along $\boldsymbol{e}_{3}$ and (ii) plane-strain simple shear. The results are compared with the predictions from other averaging schemes.
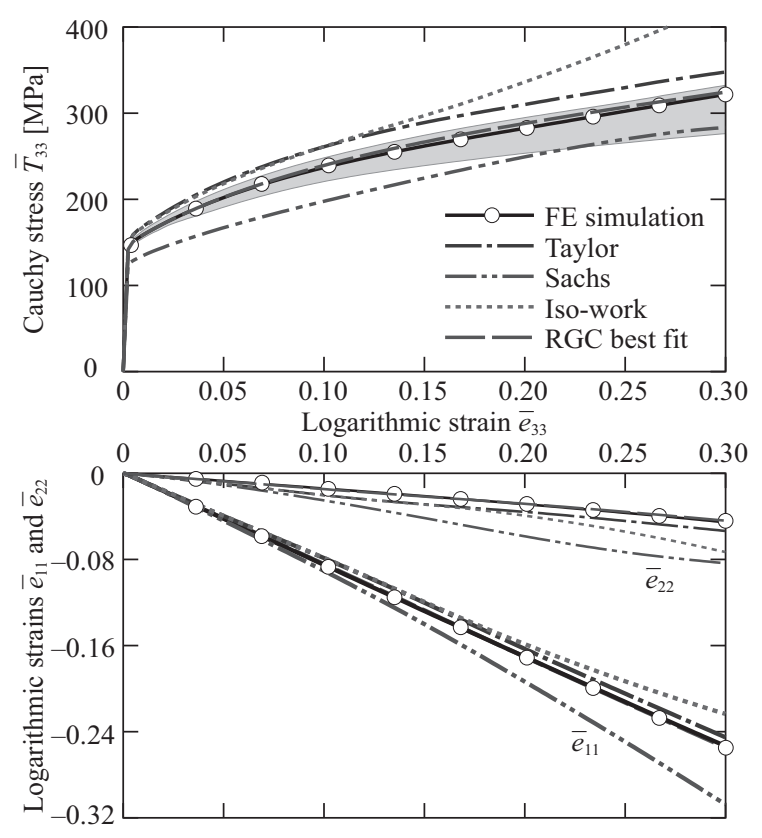

Figure 4: The effective axial stress $\bar{T}_{33}$ (top) and the lateral strains $\bar{e}_{11}$ and $\bar{e}_{22}$ (bottom) during uniaxial tension as predicted by various averaging schemes.

Fig. 4 shows the evolution of the overall axial stress and the lateral strains during uniaxial straining predicted by various averaging schemes. The grey-shaded area indicates the range of the prediction of the RGC scheme in terms of the axial stress-strain response. As shown in Fig. 4 top, the upper bound of the RGC prediction in terms of the stress-strain response, which is obtained by applying the condition of zero mismatch or fully compatible relaxation, is lower than the stress resulting from the Taylor model (uniform deformation). The lower bound of the RGC prediction is obtained by a penalty-free assumption through imposing the penalty weight $\xi_{0}=0$. In general, this lower bound is higher than the prediction of the Sachs model (uniform stress). The result of the FE simulation, where each grain is represented by 512 linear hexahedral elements, falls within the grey-shaded RGC range. This suggests that the RGC scheme gives a significantly better accuracy in comparison to the classical Taylor and Sachs bounds.

The best fit to the FE result is given by the RGC prediction with $\xi_{0}=2 \cdot 10^{-6}$ and $c_{0}=100$. In addition to the axial stress-strain response, the present RGC scheme gives a relatively good prediction in terms of the effective material anisotropy. As indicated in Fig. 4 bottom, the lateral strains predicted by the RGC scheme with the best fitted parameters are nearly identical to those resulting from FE simulation. Moreover, the present RGC scheme shows a significantly better accuracy than the iso-work weightedTaylor model [8] in both regards.

In the case of simple shear, Fig. 5 shows the overall re- 


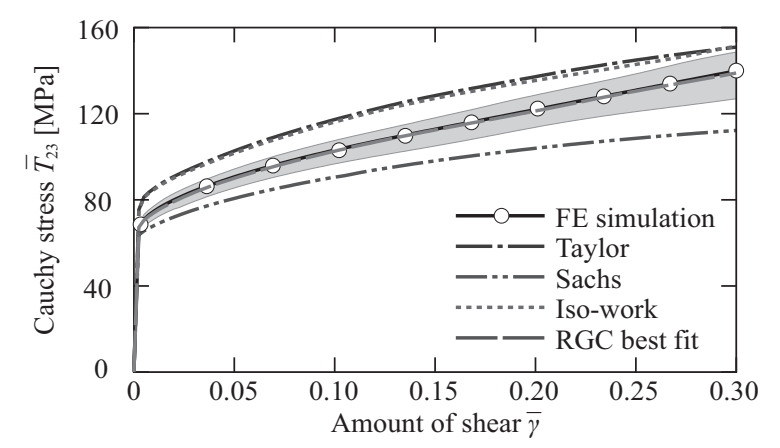

Figure 5: Prediction of different averaging schemes in terms of the effective shear stress $\bar{T}_{23}$ of the cluster undergoing simple shear.

sponse of the cluster in terms of the stress component in the shearing direction. The conclusions drawn for loading in tension equally hold in the case of simple shear. It is worth noting that the best fit to the FE simulation is achieved by the RGC scheme with the same values of $\xi_{0}$ and $c_{0}$ as those obtained for uniaxial tension.

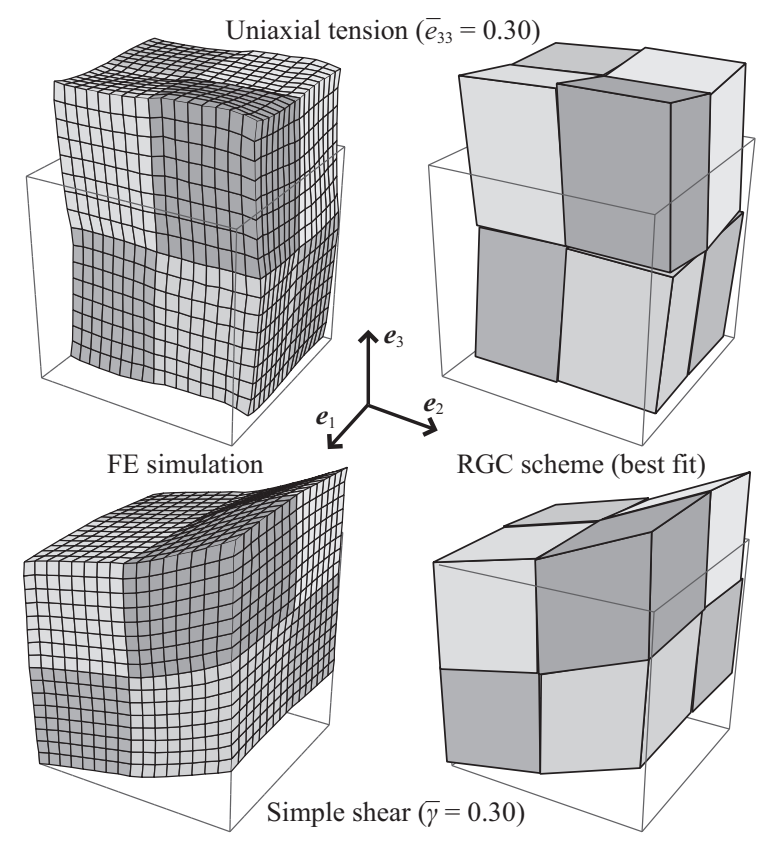

Figure 6: Comparison between (local) grain deformations calculated using FE simulations and the corresponding best fitted results using the RGC scheme.

Fig. 6 visualizes the local deformation of individual grains during uniaxial tension and simple shear obtained using FE simulations and those calculated by the RGC scheme with the best fitted parameters. The result shows that in addition to the overall response, the RGC scheme is able to mimic, up to a certain level, the detail of the FE simulation, as indicated by a strong correlation in terms of the local deformation of the individual grains between the predictions of the RGC scheme and the result of the FE simulations.

\section{CLOSING REMARKS}

The present RGC scheme shows a relatively accurate prediction in terms of the overall mechanical behavior, as well as the detailed local responses, when compared to the classical Taylor and Sachs models. This is achieved by introducing a certain additional degree of freedom (by means of interface relaxations) into the local deformation of individual grains.

Regarding computational effort, the RGC scheme requires some iterations of the Newton-Raphson procedure to obtain a convergent solution of (10), hence is about five times slower than the Taylor model, but orders of magnitude faster than the presented FE simulations.

\section{REFERENCES}

[1] M. Crumbach, M. Goerdeler, G. Gottstein, L. Neumann, H. Aretz, and R. Kopp.

Through-process texture modelling of aluminium alloys. Model. Simul. Mater. Sc., 12:S1-S18, 2004.

[2] P. Van Houtte, L. Delannay, and I. Samajdar. Quantitative prediction of cold rolling textures in low carbon steel by means of the lamel model. Texture Microstruct., 31:109-149, 1999.

[3] R.A. Lebensohn and C.N. Tomé. A self-consistent anisotropic approach for the simulation of plastic-deformation and texture development of polycrystals - application to zirconium alloys. Acta Metall. Mater., 41(9):2611-2624, 1993.

[4] V. Kouznetsova, M.G.D. Geers, and W.A.M. Brekelmans. Multi-scale constitutive modelling of heterogeneous materials with a gradient-enhanced computational homogenization scheme. Int. J. Numer. Meth. Eng., 54:1235-1260, 2002.

[5] R.A. Lebensohn. N-site modeling of a 3d viscoplastic polycrystal using fast fourier transform. Acta Mater., 49:2723-2737, 2001.

[6] D.D. Tjahjanto, P. Eisenlohr, and F. Roters. A novel grain cluster-based homogenization scheme. 2008. Submitted.

[7] S.R. Kalidindi, C.A. Bronkhorst, and L. Anand. Crystallographic texture evolution in bulk deformation processing of FCC metals. J. Mech. Phys. Solids, 40:537-569, 1992.

[8] D.D. Tjahjanto, F. Roters, and P. Eisenlohr. Iso-work-rate weighted-taylor homogenization scheme for multiphase steels assisted by transformation-induced plasticity effect. Steel Res. Int., 78(10-11):777-783, 2007. 